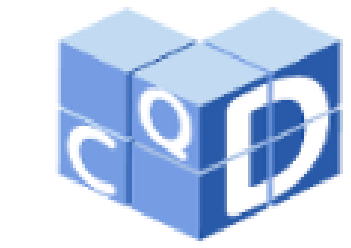

Revista Eletrônica

Paulista de Matemática

ISSN 2316-9664

Volume 17, fev. 2020

Edição Ermac

Amanda Isabela de Campos

Universidade Federal do Rio de

Janeiro

amanda.campos@coc.ufrj.br

\section{Comparação entre métodos numéricos no es- tudo de estabilidade de placas sob compressão e flexão}

Comparison of numerical methods in the stability study of plates under compression and bending

\section{Resumo}

A estabilidade estrutural de uma estrutura é garantida com a determinação da carga crítica limite, ou seja, a partir de qual carga a estrutura será instável. Este conceito é fundamental no dimensionamento estrutural de placas e para entender a influência da geometria como forma de evitar o surgimento de problemas de flambagem. As placas têm aplicações em perfis de aço, estruturas finas e esbeltas, por isso estão sujeitas a fenômenos de flambagem. As equações de equilíbrio envolvem a flexão em dois planos e a determinação de soluções exatas não é uma tarefa fácil, então, recorre-se a métodos numéricos na aproximação dessas soluções. Este trabalho, modela placas simplesmente apoiadas, sob compressão e flexão pura, e define o comportamento de flambagem, comparando soluções analíticas (que atendem as equações diferenciais parciais do fenômeno de flambagem local de placas) com soluções numéricas na aplicação do método dos elementos finitos.

Palavras-chave: Estabilidade Estrutural. Elementos finitos. Métodos Numéricos e Aplicações.

\footnotetext{
Abstract

The structural stability of a structure is guaranteed by determining the critical load limit, i.e. from which load the structure will be unstable. This concept is fundamental in the structural design of plates and to understand the influence of geometry as a way to avoid the appearance of buckling problems. The plates have applications in steel profiles and thin structures. Equilibrium equations involve bending in two planes and the determination of exact solutions is not an easy task, so numerical methods are used to approximate these solutions. This work models simply supported plates, under compression and pure bending, and defines the buckling behavior, comparing analytical solutions (which attend the partial differential equations of the local plate buckling phenomenon) with numerical solutions in the application of the finite element method.

Keywords: Structural stability. Finite elements. Numerical Methods and Applications.
} 


\section{Introdução}

Segundo Ziemian (2010) a força que gera uma mudança do estado de equilíbrio é conhecida como força crítica de flambagem de uma placa, ou seja, uma estrutura retangular de espessura pequena quando comparada as outras duas dimensões, quando sujeita a cargas superiores a crítica apresenta um mecanismo de flambagem, o que deve ser determinado para o dimensionamento de uma estrutura estável.

O fenômeno de flambagem de placas tem aplicação prática na indústria automotiva, aeroespacial e civil (BULSON, 1970; WANG, C. M.; WANG, C. Y.; REDDY, 2005) entre outras que adotam o dimensionamento de chapas de aço, ou onde é necessário um estudo de estabilidade, flambagem ou determinação de cargas críticas de painéis e placas, por exemplo.

\section{Objetivo}

O presente trabalho tem como objetivo analisar a estabilidade de diferentes placas de aço sob compressão uniforme longitudinal, e expandir a pesquisa de Campos (2019), para placas com carregamento de flexão. Para isso serão utilizados dois programas computacionais: GBTUL desenvolvido pelos professores Bebiano, Silvestre e Camotim. (2010) da Universidade Técnica de Lisboa e disponibilizado gratuitamente em seu site, o qual segue a teoria gbt (em inglês, generalized beam theory) de vigas e o programa comercial Ansys ${ }^{\circledR}$ baseado no método dos elementos finitos, já consagrado em aplicações de análises de estabilidade estrutural. Os resultados de cargas críticas com as duas ferramentas serão comparados com os resultados de soluções analíticas aproximadas de um mesmo modelo de placa.

As análises de estabilidade serão realizadas em placas de aço com largura $b=500 \mathrm{~mm}$, espessura $t=5 \mathrm{~mm}$ e comprimento variável $a=250 \mathrm{~mm}$ até $5000 \mathrm{~mm}$, para compressão. E placas de largura $b=100 \mathrm{~mm}$, espessura $t=1 \mathrm{~mm}$ e comprimento variável para flexão. As propriedades das placas adotadas estão indicadas na Tabela 1, onde $E$ é o módulo de elasticidade e $v$ coeficiente de Poisson.

Tabela 1 - Propriedades mecânicas das placas.

\begin{tabular}{cc}
$\mathbf{E}$ (MPa) & $\boldsymbol{v}$ \\
\hline 210000 & 0,3 \\
\hline
\end{tabular}

\section{Metodologia}

\subsection{Flambagem de Placas}

O modo de flambagem de uma placa retangular, simplesmente apoiada em todas as bordas com lados de comprimento $a$ e $b$, sob carregamento uniaxial, é bem representado pela flexão em dois planos. Essas condições de carga estão esquematizadas na Figura 1, onde pode-se observar, com as linhas tracejadas, o surgimento de duas semi-ondas de flambagem local na placa. 


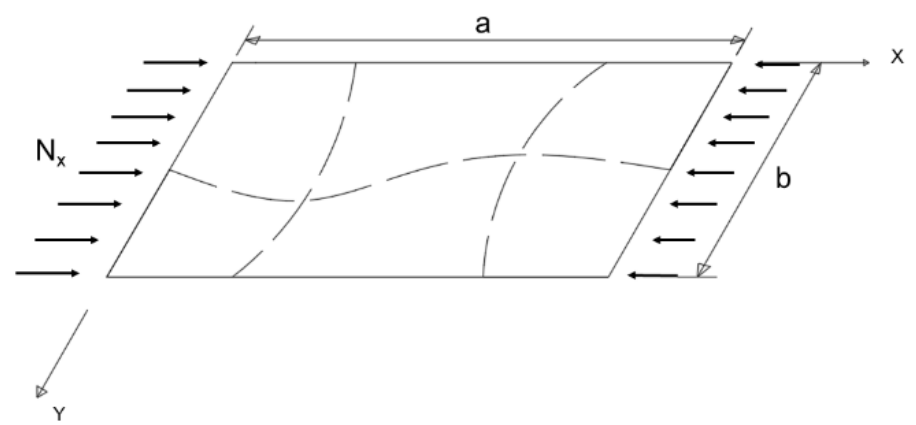

Figura 1 - Placa simplesmente apoiada com carregamento uniaxial.

Segundo os estudos de Timoshenko e Gere (1961) quando a placa está sujeita a uma carga de compressão uniforme por unidade de comprimento $\left(N_{x}\right)$ ao longo da largura, o comportamento da placa pode ser descrito por equações diferenciais parciais. A equação diferencial parcial de equilíbrio que modela a flambagem de placas com espessura constante, é indicada na Equação (1). Esse problema considera um regime de pequenas deformações e que a placa seja constituída de um material elástico linear.

$$
\frac{\partial^{4} w}{\partial x^{4}}+2 \frac{\partial^{4} w}{\partial x^{2} \partial y^{2}}+\frac{\partial^{4} w}{\partial y^{4}}+\frac{N_{x}}{D} \frac{\partial^{2} w}{\partial x^{2}}=0
$$

sendo $D=E t^{3} / 12\left(1-v^{2}\right), w$ o deslocamento fora do plano, $v$ coeficiente de Poisson, $t$ a espessura da placa e $E$ módulo de elasticidade, respectivamente.

Por se tratar de uma equação diferencial parcial existem várias funções, nesse caso de duas variáveis, que satisfazem essa igualdade. É usual sugerir funções soluções na forma de série, e ainda segundo Timoshenko e Gere (1961) uma candidata que respeita as condições de contorno desse problema é o produto das duas funções harmônicas desenvolvidas em series de Fourier. Essa proposta de solução está indicada na Equação (2).

$$
w(x, y)=\sum_{m=1}^{\infty} \sum_{n=1}^{\infty} A_{m n} \operatorname{sen}\left(\frac{m \pi x}{a}\right) \operatorname{sen}\left(\frac{n \pi y}{b}\right),
$$

onde $m$ é o número de semi-ondas na direção do comprimento (eixo $x$ ) podendo assumir os valores de $m=1,2,3, \ldots$ e $n$ é o número de semi-ondas na direção da largura, que será imposto como 1, o que gera o menor valor de carga crítica. Além disso, $A_{m n}$ são os chamados coeficiente a determinar, que dependem dos valores de $m$ e $n$ e são indicados em Timoshenko e Gere (1961) e Reis e Camotim (2001). Aplicado a função solução da Equação (2) na equação diferencial parcial do fenômeno, Equação (1), determina-se a solução não trivial do problema: tensão crítica da placa (dividindo a carga de compressão $N_{\mathrm{x}}$ obtida pela área da seção transversal). A tensão crítica é apresentada na Equação (3).

$$
\sigma_{\mathrm{cr}}=\frac{k \pi^{2} E}{12\left(1-v^{2}\right)} \frac{1}{(b / t)^{2}}
$$

onde o coeficiente de flambagem $k$ (atrelado com os chamados coeficientes a determinar) é calculado como: 


$$
k=\left(m \frac{b}{a}+\frac{1}{m} \frac{a}{b}\right)^{2},
$$

de acordo com a Equação (4), mantendo constante a largura $b$ e aumentando gradualmente o comprimento $a$, o coeficiente $k$ apresenta a variação ilustrada na Figura 2 em função da relação das dimensões da placa $a / b$. Nota-se que o valor mínimo do fator ocorre quando a placa é quadrada $a=b$ e quanto mais longa a placa o coeficiente converge para o valor de 4 , como uma placa quadrada. Então conhecendo as dimensões $a$ e $b$ da placa é possível indicar que o número de semi-ondas na direção do comprimento, ou seja, o número $m$, que a placa em flambagem exibe. A curva contínua é a curva de interesse pois são os mínimos dos coeficientes de flambagem e representa o que ocorre primeiro na placa.

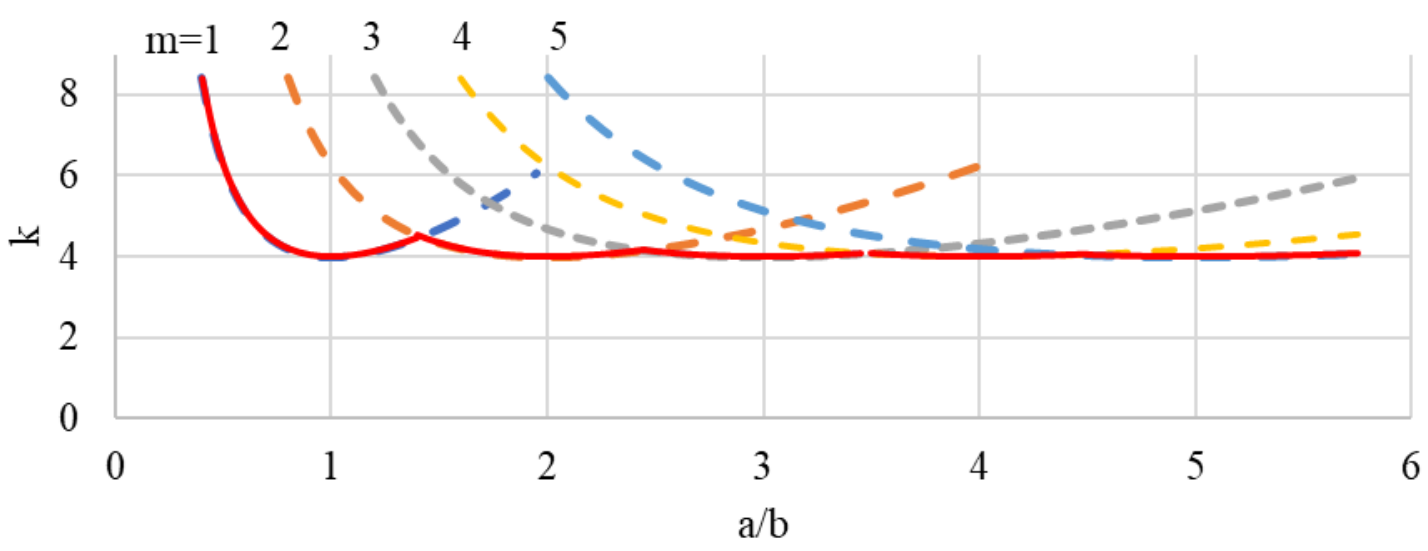

Figura 2 - Coeficiente de flambagem de placas.

O trabalho de Timoshenko e Gere (1961) complementado pelo livro de Reis e Camotim (2001) também indicam coeficientes de flambagens para outras situações de condições de contorno da placa e carregamentos diversos. Neste trabalho, foram aplicadas as condições de placa biapoiada com carregamento uniaxial.

\subsection{Método dos Elementos Finitos}

O método dos elementos finitos (MEF) é atualmente método numérico mais difundido para análise estrutural, com metodologia adotada em programas comerciais como ANSYS, ABAQUS, SAP2000 e outros. A aplicação desse método na análise de estabilidade de placas é realizada com a discretização da peça em elementos finitos de casca, onde a chapa é discretizada igualmente em toda a dimensão, ou seja, na direção transversal e longitudinal (Fig. 3).

Para o modelo numérico em elementos finitos, de placas de diferentes comprimentos, será adotado, assim como os trabalhos de Wang e Chen (2002) e Brighenti (2005), o tipo de elemento finito de casca, próprio biblioteca do programa Ansys, chamado de SHELL181, elemento de integração completa e formado por quatro nós com seis graus de liberdade em cada. As condições de contorno que representam a situação de bordos apoiados são: impedimentos aos deslocamentos nos bordos nas direções $x$ e $y$.

Testes de convergência de malha apontaram que modelos com elementos de $5 \mathrm{~mm}$ convergem para resultados coerentes. A Figura 3 representa a discretização e as condições de contorno de um modelo de placa em elementos finitos. 




Figura 3 - Discretização da malha em elementos finitos.

O MEF permite modelar qualquer condição de contorno e formato de carregamento, caracteriza-se como uma poderosa ferramenta quando são necessárias análises não lineares, considerando as imperfeições geométricas iniciais, tensões residuais e o comportamento elastoplástico do material.

Como possui um número de graus de liberdade maior que o outro método a seguir, o MEF apresenta um maior custo computacional e as desvantagens associadas à sua aplicação na análise de estabilidade de placas são: para cada comprimento de placa um novo modelo deve ser criado e uma nova análise executada, uma vez que esse tipo de método não fornece um gráfico com a variação da carga crítica em vários comprimentos; a aplicação de carregamentos e condições de contorno requer um conhecimento aprofundado do método e do programa utilizado, onde o menor erro gera um resultado discrepante.

\subsection{Teoria Generalizada de Viga (GBT)}

A Teoria Generalizada de Viga é um método de análise estrutural com potencial aplicação na análise de estabilidade de placas, representa uma extensão da teoria clássica de vigas que considera a distorção da seção transversal.

O método consiste em discretizar a forma deformada, ou seja, o modo de flambagem de uma chapa ou barra, em uma combinação linear de modos de deformação da seção transversal com variação da amplitude ao longo do comprimento. A vantagem dessa abordagem em relação ao método dos elementos finitos é que neste caso, o modelo tem menos graus de liberdade e o tempo de processamento diminui consideravelmente se comparado com o MEF, gerando um ganho computacional considerável. Como desvantagem pode-se apontar a forma deformada da peça, com uma escala monocolor, sendo impossível distinguir onde há concentração de tensões, por exemplo. Ao contrário do MEF, onde é gerado um mapa de cores da distribuição de tensões da peça deformada.

O software GBTUL aplica a teoria generalizada de viga, Bebiano et al. (2010), e é disponibilizado gratuitamente, sendo um programa de interface amigável que realiza a análise de estabilidade de placas, entretanto é um software de código fechado não admitindo a completa identificação das etapas de análise ou alterações. 


\section{Resultados}

\subsection{Compressão}

Os resultados de tensão crítica de flambagem das placas com diferentes comprimentos sob compressão estão indicados na Tabela 2, além de uma comparação entre os três métodos propostos: resultados analíticos, modelos em elementos finitos e com o auxílio do programa GBTUL. Os resultados de tensão crítica analítico foram obtidos com a equação (3) com valores variáveis de $k$, de acordo com o comprimento da placa, conforme indicado na Tabela 2.

Tabela 2 - Propriedades mecânicas e geométricas da placa.

\begin{tabular}{cccccccc}
\hline $\begin{array}{c}\text { Comprimento } \\
(\mathbf{m m})\end{array}$ & $\mathbf{m}$ & $\boldsymbol{k}$ & $\begin{array}{c}\text { Analítico } \\
\left(\mathbf{N} / \mathbf{m m}^{2}\right)\end{array}$ & $\begin{array}{c}\text { Ansys } \\
\boldsymbol{\sigma}_{\mathbf{c r}} \\
\left(\mathbf{N} / \mathbf{m m}^{2}\right)\end{array}$ & $\begin{array}{c}\text { Diferença } \\
(\boldsymbol{\%})\end{array}$ & $\begin{array}{c}\text { GBTUL } \\
\boldsymbol{\sigma}_{\mathbf{c r}} \\
\left(\mathbf{N} / \mathbf{m m}^{2}\right)\end{array}$ & $\begin{array}{c}\text { Diferença } \\
(\boldsymbol{\%})\end{array}$ \\
\hline 250 & 1 & 6.25 & 118.625 & 117.776 & $0.72 \%$ & 118.419 & $0.17 \%$ \\
500 & 1 & 4.00 & 75.920 & 75.394 & $0.69 \%$ & 75.859 & $0.08 \%$ \\
750 & 2 & 4.34 & 82.379 & 81.918 & $0.56 \%$ & 82.273 & $0.13 \%$ \\
1000 & 2 & 4.00 & 75.920 & 75.516 & $0.53 \%$ & 75.859 & $0.08 \%$ \\
1250 & 3 & 4.13 & 78.472 & 78.083 & $0.50 \%$ & 78.384 & $0.11 \%$ \\
1500 & 3 & 4.00 & 75.920 & 75.557 & $0.48 \%$ & 75.859 & $0.08 \%$ \\
1750 & 4 & 4.07 & 77.282 & 76.924 & $0.46 \%$ & 77.201 & $0.10 \%$ \\
2000 & 4 & 4.00 & 75.920 & 75.577 & $0.45 \%$ & 75.859 & $0.08 \%$ \\
2250 & 5 & 4.04 & 76.766 & 76.425 & $0.44 \%$ & 76.690 & $0.10 \%$ \\
2500 & 5 & 4.00 & 75.920 & 75.590 & $0.43 \%$ & 75.859 & $0.08 \%$ \\
2750 & 6 & 4.03 & 76.496 & 76.166 & $0.43 \%$ & 76.423 & $0.10 \%$ \\
3000 & 6 & 4.00 & 75.920 & 75.598 & $0.42 \%$ & 75.859 & $0.08 \%$ \\
3250 & 7 & 4.02 & 76.338 & 76.015 & $0.42 \%$ & 76.266 & $0.09 \%$ \\
3500 & 7 & 4.00 & 75.920 & 75.604 & $0.42 \%$ & 75.859 & $0.08 \%$ \\
3750 & 8 & 4.02 & 76.237 & 75.920 & $0.42 \%$ & 76.166 & $0.09 \%$ \\
4000 & 8 & 4.00 & 75.920 & 75.608 & $0.41 \%$ & 75.859 & $0.08 \%$ \\
4250 & 9 & 4.01 & 76.168 & 75.856 & $0.41 \%$ & 76.099 & $0.09 \%$ \\
4500 & 9 & 4.00 & 75.920 & 75.611 & $0.41 \%$ & 75.859 & $0.08 \%$ \\
4750 & 10 & 4.01 & 76.120 & 75.81 & $0.41 \%$ & 76.051 & $0.09 \%$ \\
5000 & 10 & 4.00 & 75.920 & 75.614 & $0.40 \%$ & 75.859 & $0.08 \%$ \\
\hline
\end{tabular}

A diferença percentual entre as cargas críticas analíticas e em elementos finitos foram em média $0,43 \%$ e pode-se observar que essa diferença é maior em placas de comprimentos menores, onde as condições de contorno têm maior influência nos resultados. Essa diferença deve-se a forma de imposição das condições de contorno, porém como as diferenças são sempre inferiores a 1\%, esse "erro" é considerado irrelevante. Já a diferença entre o analítico e o programa GBTUL é menor que $0,1 \%$ o que é um erro mínimo então pode-se afirmar que o modelo foi validado para análises de cargas críticas.

A variação da tensão crítica com o comprimento se comporta como esperado uma vez que o coeficiente de flambagem $k$, como dito, converge para o valor de 4 em placas longas, dessa forma o valor da tensão crítica determinada com o Ansys tende para o valor obtido ana- 
liticamente com $k=4$, que é $\sigma_{\mathrm{cr}}=75,920 \mathrm{kN}$, determinada com a Equação (3). Isso é observado na Figura 4.

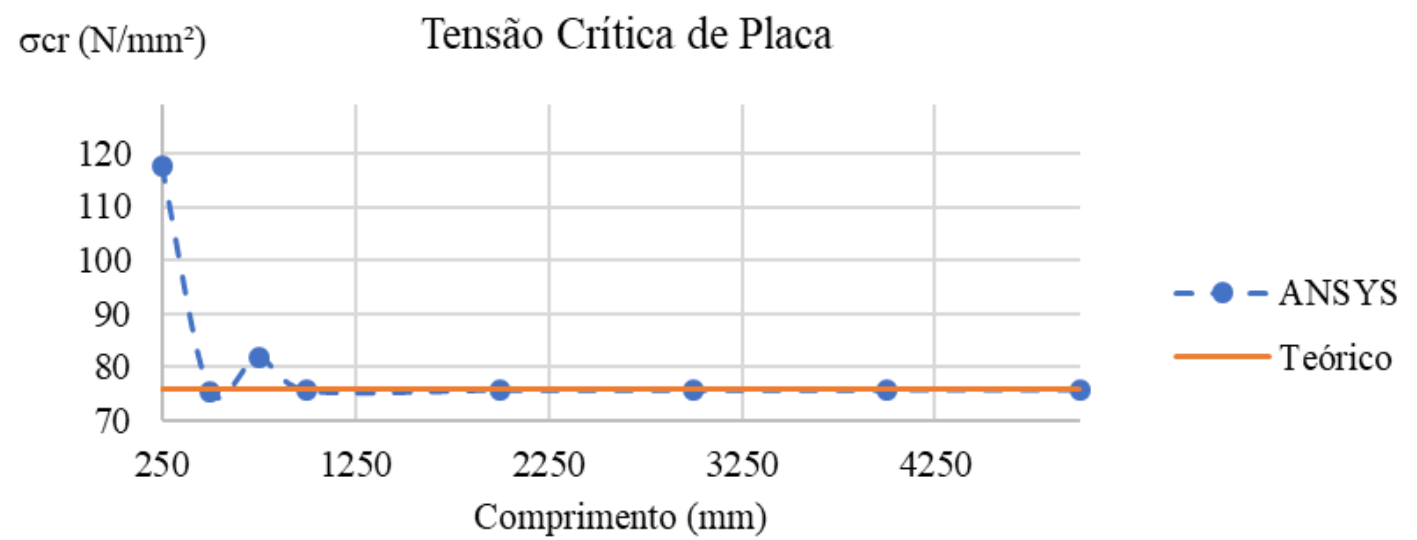

Figura 4 - Comparação entre tensões críticas sob compressão.

Os modos de flambagem de alguns comprimentos de placas, obtidos com o programa Ansys, estão exibidos na Figura 5.1 e os modos obtidos com o programa GBTUL na Figura 5.2. Com a forma dos comprimentos de ondas, nota-se que cada onda tem o mesmo comprimento da largura da placa como esperado e o número de ondas aumenta com o aumento do comprimento da placa na proporção imposta pela Equação (4). Escala de cores normalizada, onde o vermelho é o deslocamento unitário e máximo.



Figura 5.1 - Modos de flambagem de placas com aumento do comprimento sob compressão pura (MEF).

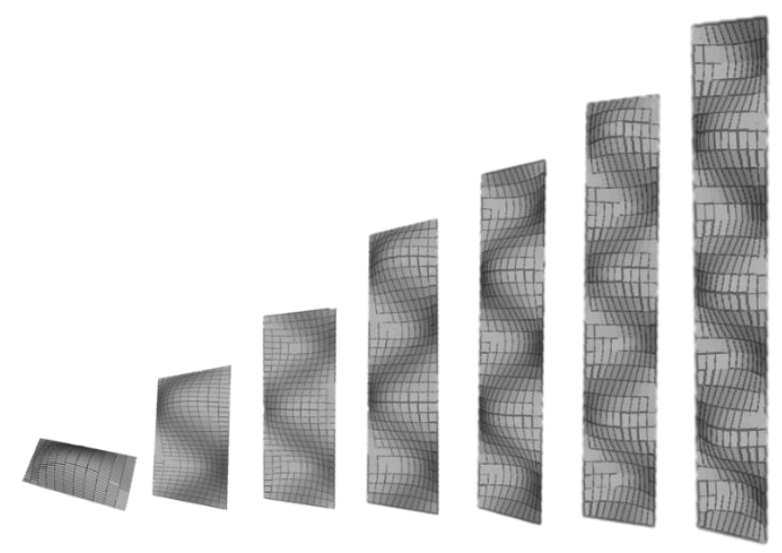

Figura 5.2 - Modos de flambagem de placas com aumento do comprimento sob compressão pura (GBTUL).

PRONI, C.; HANEDA, R. N.; FILLETTI, É. R. Comparação entre métodos numéricos no estudo de estabilidade de placas sob compressão e flexão. C.Q.D.Revista Eletrônica Paulista de Matemática, Bauru, v. 17, p. 206-215, fev. 2020. Edição Ermac. 


\subsection{Flexão}

O modelo de placa retangular, largura de $b=100 \mathrm{~mm}$ e espessura de $t=1 \mathrm{~mm}$, quando submetida a flexão pura, como esquematizado na Figura 6, com dois bordos biapoiados, e, $x=0$ e $x=\mathrm{a}$, foi submetido a análise de flambagem elástica com os programas Ansys e GBTUL.

O carregamento de flexão pura é aplicado a partir de forças distribuídas ao longo do bordo com intensidade no formato indicado na Figura 6. O valor dessa força $\left(N_{x}\right)$ é variável ao longo da altura y e esse pode ser calculado com a Equação (5), expressão essa adotada para modelar as cargas no modelo em elementos finitos.

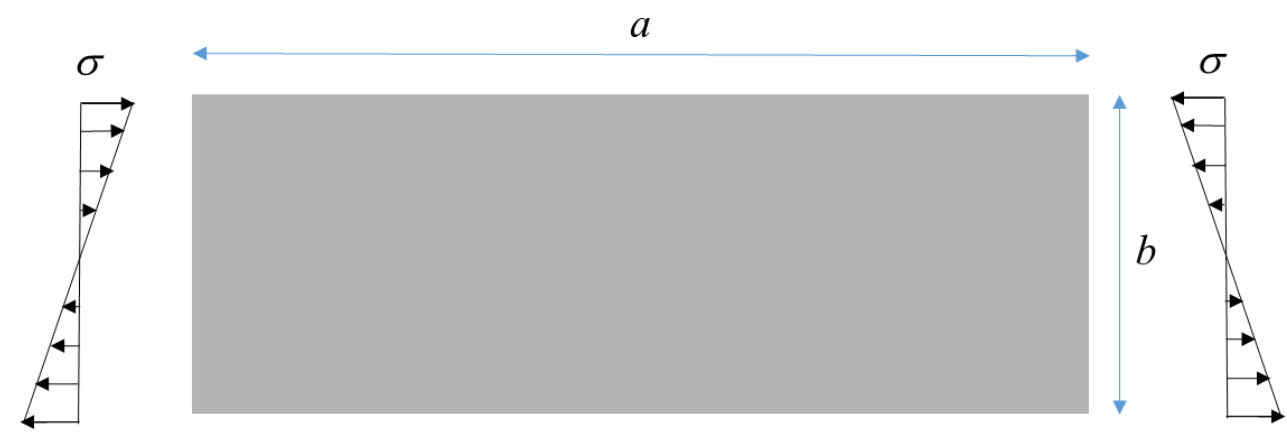

Figura 6 - Modelo de placa submetida a flexão pura

$$
N_{x}=N_{0}\left(1-\alpha \frac{y}{b}\right)
$$

A deflexão $w$ da placa quando submetida a força crítica flambagem, segue a mesma equação da compressão, Equação (2), de forma que, segundo Timoshenko e Gere (1961), a tensão crítica para uma placa sob flexão pura também pode ser calculada analiticamente com a Equação (3), porém como o coeficiente de flambagem $k$ depende do carregamento e das condições de contorno, nesse caso de flexão pura e placa simplesmente apoiada, valores diferentes são recomendados, e dependendo da relação entre as dimensões largura e comprimento da placa, esses valores são em média $k \cong 39,7$.

Tabela 3 aponta a comparação entre as tensões críticas de flambagem para diferentes comprimentos de placas, quando submetidas a flexão pura, com os dois métodos numéricos e a expressão analítica.

Tabela 3 - Propriedades mecânicas e geométricas da placa.

\begin{tabular}{|c|c|c|c|c|c|c|c|c|}
\hline $\begin{array}{c}\text { Comprimento } \\
a(\mathrm{~mm})\end{array}$ & $\mathbf{m}$ & $\mathbf{a} / \mathbf{b}$ & $\mathbf{k}$ & $\begin{array}{l}\text { Analítico } \\
\left(\mathrm{N} / \mathbf{m m}^{2}\right)\end{array}$ & $\begin{array}{c}\text { Ansys } \\
\sigma_{c r} \\
\left(\mathbf{N} / \mathbf{m m}^{2}\right)\end{array}$ & $\begin{array}{c}\text { Diferença } \\
(\%)\end{array}$ & $\begin{array}{c}\text { GBTUL } \\
\sigma_{\mathrm{cr}} \\
\left(\mathrm{N} / \mathbf{m m}^{2}\right)\end{array}$ & $\begin{array}{c}\text { Diferença } \\
(\%)\end{array}$ \\
\hline 100 & 2 & 1,0 & 43,8 & 831,32 & 818,88 & $1,50 \%$ & 801,78 & $3,55 \%$ \\
\hline 250 & 4 & 2,5 & 40,7 & 772,49 & 768,90 & $0,46 \%$ & 754,29 & $2,36 \%$ \\
\hline 500 & 7 & 5,0 & 39,7 & 753,51 & 768,12 & $1,94 \%$ & 753,93 & $0,06 \%$ \\
\hline 650 & 10 & 6,5 & 39,7 & 753,51 & 766,75 & $1,76 \%$ & 752,00 & $0,20 \%$ \\
\hline
\end{tabular}


Os modos de flambagem de alguns comprimentos de placas, obtidos com o programa Ansys, estão exibidos na Figura 7.1 e os modos obtidos com o programa GBTUL estão indicados na Figura 7.2. Nota-se que o número de ondas aumenta com o aumento do comprimento da placa. A escala de cores está normalizada, onde o vermelho é o deslocamento unitário e máximo.

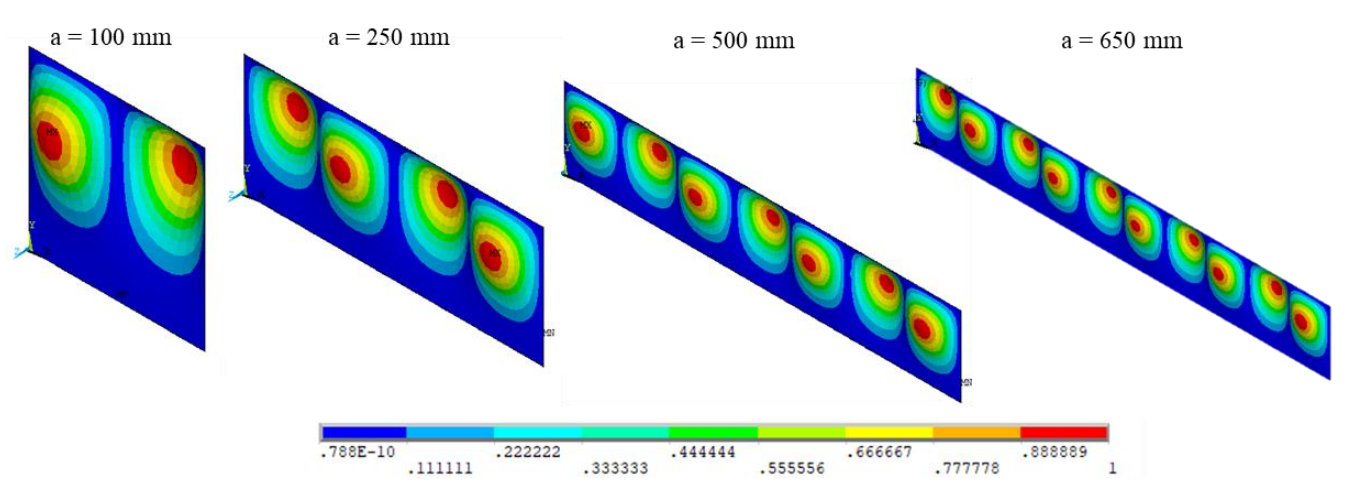

Figura 7.1 - Modos de flambagem de placas com aumento do comprimento sob flexão pura (MEF).
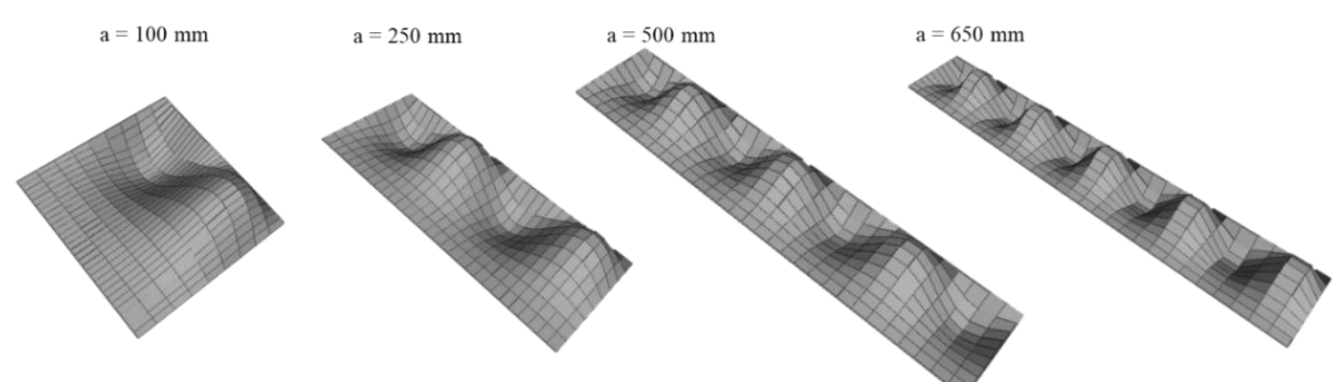

Figura 7.2 - Modos de flambagem de placas com aumento do comprimento sob flexão pura (GBTUL).

Os valores de tensão crítica analíticos e numéricos tendem a convergir para placas longas, o que é observado com o aumento do comprimento da placa, isso porque o modelo passa a ter menos influência das condições de contorno.

Os modos de flambagem obtidos com os dois modelos (Fig. 7.1 e 7.2) são praticamente iguais e comparando com as cargas crítica de flambagem com as obtidas analiticamente, encontra-se diferenças menores que $3 \%$, então, pode-se afirmar que os modelos estão validados e podem ser adotados para quaisquer comprimentos de placa sob flexão pura.

\section{Conclusões}

Como não existe solução exata analítica para o problema de estabilidade é necessário recorrer a métodos numéricos de discretização do meio continuo, ou seja, o método analítico exato é inviável e, portanto, requer uma aproximação em séries de Fourier, como a solução analítica proposta neste trabalho ou então modelos numéricos em elementos finitos, por exemplo. Para validar e comprovar esses modelos foi feito um estudo a partir da manipulação geométrica de placas, sujeitas a compressão simples e flexão pura e então, comparados os resultados de análise de estabilidade das duas ferramentas numéricas.

Os modos de flambagem das Figuras 5.1 e 5.2 apresentaram o número de semi-ondas esperado de acordo com a suas dimensões e o comportamento do coeficiente de flambagem 
ilustrado na Figura 2. Ou seja, para uma placa de comprimento $a=1500 \mathrm{~mm}$ a relação $a / b$ será 3, o que de acordo com a Figura 2 garante um número de semi-ondas $\mathrm{m}=3$, o que é observado no modo de flambagem da terceira imagem da deformada do programa Ansys (Figura 5.1). Além de que o valor da carga crítica foi validado o programa livre GBTUL.

Com os métodos propostos, portanto é possível obter um projeto simples com fórmulas de aproximações analíticas ou modelos numéricos que podem ser aplicados satisfatoriamente em análises de estabilidade de placas no sentido de evitar que fenômenos de flambagem ocorram.

Para a análise de flambagem com aplicação de compressão e flexão de placas, o MEF não é a abordagem mais vantajosa, recomenda-se então, para esse tipo de problema, a preferência pelo programa GBTUL, que fornece um gráfico com a variação da carga crítica em vários comprimentos e o gasto computacional é menor, gerando resultados mais rápidos e com diferenças insignificantes.

\section{Referências}

BEBIANO, R.; SILVESTRE, N.; CAMOTIM, D. GBT theoretical background. Lisbon: DECivil/IST, 2010. Disponível em: http://www.civil.ist.utl.pt/gbt/wpcontent/uploads/2013 /10/GBT - TheoreticalBackgound.pdf. Acesso em: 2 abr. 2019.

BRIGHENTI, R. Numerical buckling analysis of compressed or tensioned cracked thin plates. Engineering Structures, v. 27, n. 2, p. 265-276, 2005.

BULSON, P. S. The stability of flat plates. London: Chatto and Windus, 1970.

CAMPOS, A. I. Comparação entre métodos numéricos no estudo de estabilidade de placas sob compressão. In: ENCONTRO REGIONAL DE MATEMATICA APLICADA E COMPUTACIONAL, 6., 2019, Bauru. Caderno de trabalhos completos e resumos [...]. Bauru: Unesp, Faculdade de Ciências, 2019. p. 105-110. Disponível em: https://drive. google.com /file/d/10TKZOqD7_VB-8GMRKUCAMATYMjkDu5Gu/view/. Acesso em: 18 ago. 2019.

REIS, A.; CAMOTIM, D. Estabilidade estrutural. Amadora: McGraw-Hill de Portugal, 2001.

TIMOSHENKO, S.; GERE, J. Theory of elastic stability. 2nd ed. New York: McGraw-Hill, 1961.

WANG, C. M.; CHEN, Y. Elastic/plastic buckling of simply supported, rectangular plates under intermediate and end uniaxial loads, In: KKHTCNN SYMPOSIUM ON CIVIL ENGINEERING, 15., 2002, Singapore. Proceedings [...]. Singapore: KAIST, 2002. p. 89-99.

WANG, C. M.; WANG, C. Y.; REDDY, J. N. Exact solutions for buckling of structural members. Flórida: CRC Press, 2005.

ZIEMIAN, R. D. (ed.). Guide to stability design criteria for metal structures. Nova Jersey: John Wiley \& Sons, 2010. 